\title{
Resistance to TRAIL in mantle cell lymphoma cells is associated with the decreased expression of purine metabolism enzymes
}

\author{
JANA POSPISILOVA ${ }^{1}$, ONDREJ VIT ${ }^{1}$, LUCIE LORKOVA ${ }^{1}$, MAGDALENA KLANOVA ${ }^{1}$, \\ JAN ZIVNY $^{1}$, PAVEL KLENER ${ }^{1}$ and JIRI PETRAK ${ }^{1,2}$ \\ ${ }^{1}$ Institute of Pathological Physiology, First Faculty of Medicine, Charles University in Prague, 12853 Prague; \\ ${ }^{2}$ Institute of Hematology and Blood Transfusion, 12820 Prague, Czech Republic
}

Received January 25, 2013; Accepted March 1, 2013

DOI: $10.3892 / \mathrm{ijmm} .2013 .1302$

\begin{abstract}
Mantle cell lymphoma (MCL) is a rare aggressive type of B-cell non-Hodgkin's lymphoma. Response to chemotherapy tends to be short and virtually all patients sooner or later relapse. The prognosis of relapsed patients is extremely poor. The tumor necrosis factor-related apoptosis-inducing ligand (TRAIL) is considered one of the novel experimental molecules with strong antitumor effects. TRAIL triggers extrinsic apoptotis in tumor cells by binding to TRAIL 'death receptors' on the cell surface. Recombinant TRAIL has shown promising pro-apoptotic effects in a variety of malignancies including lymphoma. However, as with other drugs, lymphoma cells can develop resistance to TRAIL. Therefore, the aim of this study was to identify the molecular mechanisms responsible for, and associated with TRAIL resistance in MCL cells. If identified, these features may be used as molecular targets for the effective elimination of TRAIL-resistant lymphoma cells. From an established TRAIL-sensitive mantle cell lymphoma cell line (HBL-2) we derived a TRAIL-resistant HBL-2/R subclone. By TRAIL receptor analysis and differential proteomic analysis of HBL-2 and HBL-2/R cells we revealed a marked downregulation of all TRAIL receptors and, among others, the decreased expression of 3 key enzymes of purine nucleotide metabolism, namely purine nucleoside phosphorylase, adenine phosphoribosyltransferase and inosine-5'-monophosphate dehydrogenase 2 , in the resistant HBL-2/R cells. The downregulation of the 3 key enzymes of purine metabolism can have profound effects on nucleotide homeostasis in TRAIL-resistant lymphoma cells and can render such cells vulnerable to any further disruption of purine nucleotide metabolism. This pathway represents a 'weakness' of the TRAIL-resistant MCL cells and has potential as a therapeutic target for the selective elimination of such cells.
\end{abstract}

Correspondence to: Ms. Jana Pospisilova, Institute of Pathological Physiology, First Faculty of Medicine, Charles University in Prague, U Nemocnice 5, 12853 Prague, Czech Republic

E-mail: jana.pospisilova@lf1.cuni.cz

Key words: mantle cell lymphoma, TRAIL, drug resistance, purine nucleotide metabolism, proteomics

\section{Introduction}

Mantle cell lymphoma (MCL) is a rare agressive type of B-cell non-Hodgkin's lymphoma with an estimated annual incidence in Europe of 0.45/100,000 individuals (1). MCL is a biologically and clinically heterogeneous disease; the immunophenotype of neoplastic cells reflects the phenotype of cells similar to lymphocytes in the mantle zone of normal germinal follicles (2). The genetic hallmark of MCL cells is a translocation between chromosomes 11 and 14, t(11;14)(q13;q32), juxtaposing the gene for immunoglobulin heavy chain and the gene encoding cyclin D1. This results in cyclin D1 overexpression $(3,4)$.

The standard of care for newly diagnosed MCL patients is combined immunochemotherapy alternating rituximab-CHOP (R-CHOP; cyclophosphamide, vincristine, doxorubicin and prednisone) and R-HDAC (high-dose cytarabine). The addition of rituximab and HDAC to CHOP has improved the survival of MCL patients in the last 2 decades from 3 to 5 years. However, the response to therapy tends to be short and virtually all patients sooner or later relapse. There is no standard of care for relapsed or refractory MCL patients. Salvage therapy usually comprises diverse regimens based on fludarabine, gemcitabine, cisplatin, bendamustine, bortezomib (inhibitor of $26 \mathrm{~S}$ proteasome) or temsirolimus (inhibitor of mTOR). Recently, several new experimental molecules have shown promise in the therapy of relapsed or resistant MCL, including lenalidomide (immunomodulatory agent), ibrutinib (PCI-32765, inhibitor of Bruton's tyrosine-kinase), new monoclonal antibodies (e.g., anti-CD20 ofatumumab), as well as other agents (5). Combination therapies are currently being evaluated in clinical trials; however, novel drugs are required.

The tumor necrosis factor-related apoptosis-inducing ligand (TRAIL) is considered one of the novel experimental molecules with strong antitumor effects. TRAIL is a type II transmembrane protein from the tumor necrosis factor superfamily $(6,7)$ expressed mostly by cells of the immune system (natural killer cells, cytotoxic T-cells, macrophages and dendritic cells). The main function of this molecule is thought to be in tumor immunosurveillance, but its actual molecular role remains to be elucidated.

TRAIL can trigger extrinsic apoptotis in target cells by binding to TRAIL death receptors located on the cell surface (8). This interaction is performed by a long extracellular C-terminal 
region of the TRAIL molecule. There are 4 distinct cell surface TRAIL receptors in humans (DcR1, DcR2, DR4 and DR5) encoded by separate genes $(9,10)$. However, only DR4 and DR5 contain a functional death domain (structurally conserved protein interaction domain) and are capable of signaling apoptosis. Two decoy receptors (DcR1 and DcR2) lack a functional death domain and inhibit TRAIL signaling by competing with death receptors for TRAIL $(9,10)$. The binding of TRAIL to DR4 or DR5 leads to receptor homotrimerization and formation of the death-inducing signaling complex (DISC) (11). Through the DISC a caspase machinery is activated, which results in apoptosis (12). TRAIL death receptors DR4 and DR5 are ubiquitously expressed, indicating that most tissues and cell types are potential targets of TRAIL signaling (13). Nevertheless, TRAIL seems to induce apoptosis only in tumor cells but not in healthy tissues. Due to its selective pro-apoptotic effect, TRAIL has attracted much attention for its possible use in cancer therapy. In vitro, a recombinant soluble TRAIL molecule has shown cytostatic or cytotoxic effects in a wide variety of tumor cell lines, including leukemia and lymphoma cells, but not in normal cells $(6,7,10,11,14-19)$. The administration of a recombinant soluble TRAIL molecule has been shown to induce the regression or complete remission of tumors in tumor xenograft models (11,20-26). The efficacy of recombinant TRAIL and agonistic antibodies recognizing either receptor DR4 or DR5 has been investigated in numerous clinical trials, as recently reviewed (27).

TRAIL has also shown promising pro-apoptotic effects in a variety of lymphoma cell lines including MCL (15). However, as with other drugs, cancer cells can develop resistance to TRAIL following prolonged exposure to sublethal doses of TRAIL $(14,28)$. Resistance to TRAIL-mediated apoptosis can arise due to changes at the cell membrane level (typically by loss of expression or mutation of functional DR4 and/or DR5 at the cell surface) or on the intracellular level (such as incorrect formation of DISC and abberant expression of caspases) (29). The successful therapy of malignancies in general, and particularly those with very poor prognosis, such as MCL, depends on the effective management of drug resistance. An in-depth understanding of the processes involved in the development of drug resistance and a detailed description of secondary molecular changes associated with resistance are essential for successful cancer therapy. Specific molecular changes which occur in drug-resistant cells can confer a potential selective disadvatage to such cells and may be used as targets for the effective elimination of drug-resistant lymphoma cells.

The aim of this study was to elucidate the molecular mechanisms responsible for TRAIL resistance in MCL cells, as well as the secondary molecular alterations associated with this process. We also aimed to identify the phenotypic features specific for TRAIL-resistant MCL cells. If identified, these molecular features can be, at least theoretically, used as molecular targets for the effective elimination of TRAILresistant lymphoma cells in experimental therapies.

\section{Materials and methods}

Cell growth and cellular toxicity assay. HBL-2 cells were grown in Iscove's modified Dulbecco's medium in the presence of $10 \%$ foetal bovine serum, $1 \%$ penicillin-streptomycin solution in a $37^{\circ} \mathrm{C}$ humidified atmosphere with $5 \% \mathrm{CO}_{2}$. TRAILresistant $\mathrm{HBL}-2 / \mathrm{R}$ cells were derived by selective pressure of increasing concentrations of human recombinant TRAIL (Apronex Biotechnologies, Czech Republic) up to $1,000 \mathrm{ng} / \mathrm{ml}$ in medium from the wild-type HBL-2 cells in 5 weeks. The toxicity of TRAIL to HBL-2 and HBL-2/R was measured using the colorimetric WST-8-based Quick Cell proliferation Assay kit II (BioVision, San Francisco, CA, USA) according to the manufacturer's instructions. Briefly, 40,000 cells were seeded in a 96-well plate in $300 \mu \mathrm{l}$ of medium supplemented with increased concentrations of TRAIL up to $1,000 \mathrm{ng} / \mathrm{ml}$ in medium for 1-4 days. After the addition of WST reagent, absorbance was measured on a Sunrise microplate absorbance reader (Tecan Group Ltd., Männedorf, Switzerland) with a $450 \mathrm{~nm}$ reading filter and $630 \mathrm{~nm}$ reference filter. The absorbance of free medium was used as the background level, triplicate samples were grown and measured for each cell type and TRAIL concentration. Mean values were calculated. All chemicals were purchased from Sigma-Aldrich (St. Louis, MO, USA) unless specified otherwise.

Flow cytometric analysis. HBL-2 and HBL-2/R cells $\left(2 \times 10^{5}\right.$ cells for each assay) were washed in PBS buffer $(0.5 \%$ foetal bovine serum in PBS), stained with phycoerythrin-conjugated antibodies against TRAIL receptors DR4, DR5, DcR1 and DcR2 (anti-hTRAIL R1, anti-hTRAIL R2, anti-hTRAIL R3 and anti-hTRAIL R4; R\&D Systems, Minneapolis, MN, USA) and analyzed by flow cytometry in triplicate (FASCCanto II, BD Biosciences, San Jose, CA, USA). Unstained cells and cells incubated with isotype controls served as the background fluorescence controls.

Sample preparation for two-dimensional electrophoresis. HBL-2 and HBL-2/R cells $\left(6 \times 10^{7}\right)$ were harvested, washed twice with PBS and cell pellets were frozen and stored at $-80^{\circ} \mathrm{C}$. Samples were thawed and homogenized in lysis buffer [7 $\mathrm{M}$ urea, $2 \mathrm{M}$ thiourea, 4\% CHAPS, $60 \mathrm{mM}$ dithiothreitol (DTT) and $1 \%$ ampholytes (Bio-Lyte 3-10 Buffer, Bio-Rad, Hercules, CA, USA)] and protease inhibitor cocktail (Roche Diagnostics $\mathrm{GmbH}$, Mannheim, Germany) for $20 \mathrm{~min}$ at room temperature with occasional vortexing. Samples were sedimented at $18,000 \mathrm{x} \mathrm{g}$ for $20 \mathrm{~min}$ at room temperature, supernatants were collected and protein concentration was determined by the Bradford assay (Bio-Rad). Protein concentrations in all samples were equalized to $3.3 \mathrm{mg} / \mathrm{ml}$ by dilution with lysis buffer.

Two-dimensional electrophoresis. IPG strips ( $\mathrm{pH} 4.0-7.0,24 \mathrm{~cm}$; ReadyStrip, Bio-Rad) were rehydrated overnight in $450 \mu \mathrm{l}$ of sample, representing $1.5 \mathrm{mg}$ of protein. Isoelectric focusing was performed for $70 \mathrm{kVh}$, with maximum voltage not exceeding $5 \mathrm{kV}$, current limited to $50 \mu \mathrm{A}$ per strip and temperature set to $20^{\circ} \mathrm{C}$ (Protean IEF Cell, Bio-Rad). Six replicates were run for each cell type. Focused strips were briefly rinsed in deionized water, equilibrated and reduced in equilibration buffer supplemented with DTT (6 M urea, $50 \mathrm{mM}$ Tris pH 8.8, 30\% glycerol, $2 \% \mathrm{SDS}$ and $450 \mathrm{mg}$ DTT per $50 \mathrm{ml}$ ) for $15 \mathrm{~min}$ and then alkylated in equilibration buffer with iodacetamide $(1.125 \mathrm{mg}$ iodacetamide per $50 \mathrm{ml}$ of the buffer). Equilibrated strips were then secured on $10 \%$ SDS-PAGE and secured in place by molten agarose. SDS-PAGE electrophoresis was performed in 
a Tris-glycine-SDS system using a 12-gel Protean Dodeca Cell apparatus (Bio-Rad) with buffer circulation and external cooling $\left(20^{\circ} \mathrm{C}\right)$. Gels were run at a constant voltage of $45 \mathrm{~V}$ per gel for $30 \mathrm{~min}$ and then at a constant voltage of $200 \mathrm{~V}$ for $6 \mathrm{~h}$. Gels were washed 3 times for $15 \mathrm{~min}$ in deionized water to remove redundant SDS. Gels were then stained with colloidal Coomassie Brilliant Blue (SimplyBlue ${ }^{\mathrm{TM}}$ Safestain, Invitrogen, Carlsbad, CA, USA) overnight and briefly de-stained in deionized water.

Gel image analysis and extraction of peptides. Stained gels were scanned with GS 800 calibrated densitometer (Bio-Rad) and image analysis was performed with Progenesis ${ }^{\mathrm{TM}}$ software (Nonlinear Dynamics, Ltd., Newcastle upon Tyne, UK) in semi-manual mode with 6 gel replicates for each cell type. Normalization of gel images was based on total spot density, and integrated spot density values (spot volumes) were then calculated after background subtraction. Average spot volume values (averages from the all 6 gels in the group) for each spot were compared between the groups. Protein spots were considered differentially expressed if their average normalized spot volume difference was $>1.5$-fold. As determined by the Student's t-test, a p-value $<0.05$ was considered to indicate a statistically significant difference.

Protein digestion and peptide extraction. Spots containing differentially expressed proteins were excised from the gels, cut into small pieces and washed 3 times with $25 \mathrm{mM}$ ammonium bicarbonate in $50 \%$ acetonitrile (ACN). The gels were then dried in a SpeedVac Concentrator (Eppendorf, Hamburg, Germany). Sequencing grade modified trypsin (Promega, Madison, WI, USA) (6 ng/ $\mu \mathrm{l}$ of trypsin in $25 \mathrm{mM}$ ammonium bicarbonate in $5 \%$ ACN) was added. Following overnight incubation at $37^{\circ} \mathrm{C}$, the resulting peptides were extracted with $50 \% \mathrm{ACN}$.

Matrix-assisted laser desorption/ionization-time of flight mass spectrometry (MALDI-TOF MS) and identification of selected proteins. Peptide samples were spotted on a polished steel target plate (Bruker Daltonics, Bremen, Germany) and allowed to dry at room temperature. Matrix solution (3 mg $\alpha$-cyano-4hydroxycinnamic acid in $1 \mathrm{ml}$ of $50 \%$ ACN containing $0.1 \%$ trifluoroacetic acid) was then added. MS was performed on an Autoflex II MALDI-TOF/TOF mass spectrometer (Bruker Daltonics) using a solid nitrogen laser $(337 \mathrm{~nm})$ and FlexControl software (Bruker Daltonics) in reflectron mode with positive ion mass spectra detection. The mass spectrometer was externally calibrated with Peptide Calibration Standard II (Bruker Daltonics). Spectra were acquired in the mass range 800-4,000 Da. The peak lists were generated using FlexAnalysis and searched against Swiss-Prot (2011 version, 524420 sequences) using Mascot software. The peptide mass tolerance was set to $50 \mathrm{ppm}$, taxonomy Homo sapiens, missed cleavage was set to 2, fixed modification for cysteine carbamidomethylation, and variable modifications for methionine oxidation and protein N-terminal acetylation.

Western blot analysis. Cells were lysed in NHT buffer (140 mM NaCl, 10 mM HEPES, 1.5\% Triton X-100, pH 7.4). Protein concentration in the collected supernatants was determined by the Bradford assay (Bio-Rad). Lysate samples (25-70 $\mu \mathrm{g})$ were combined with SDS loading buffer containing 2-mercaptoethanol and boiled for $5 \mathrm{~min}$. Triplicate samples were separated on $12 \%$ SDS-PAGE minigels in Tris-glycine buffer (Bio-Rad). Electrophoresis was performed at a constant voltage for $30 \mathrm{~min}$ at $45 \mathrm{~V}$ per gel, and then at $90 \mathrm{~V}$ per gel until the dye front reached the gel bottom. Proteins were transferred onto $0.45 \mu \mathrm{m}$ PVDF membranes (Milipore, Billerica, MA, USA) in a semi-dry blotter (Hoefer, San Francisco, CA, USA) at $0.8 \mathrm{~mA} / \mathrm{cm}^{2}$ of membrane. Membranes were incubated with blocking buffer containing PBS (Invitrogen), 0.1\% Tween-20 and $5 \%$ non-fat dried milk for $1 \mathrm{~h}$. As primary antibodies anti-adenine phosphoribosyltransferase (APRT; 1:1,000, rabbit polyclonal antibody), anti-purine nucleoside phosphorylase (PNP; 1:1,000, mouse monoclonal antibody) and anti-GAPDH (1:10,000, rabbit polyclonal antibody) were used. After thoroughly washing in blocking buffer, a secondary horseradish peroxidase-conjugated anti-mouse or anti-rabbit antibody was added (1:10,000). GAPDH was used as the loading control. The signal was detected using Western Blotting Luminol Reagent (Santa Cruz Biotechnology, Inc., Santa Cruz, CA, USA) and membranes were exposed to X-ray films (Kodak, Rochester, NY, USA). All used antibodies were from Santa Cruz Biotechnology.

\section{Results}

Molecular changes associated with the generation of drugresistant cells can confer potential selective disadvantage. Such a 'weakness' may be used as druggable target for effective elimination of drug-resistant lymphoma cells. Our aim was to elucidate the molecular changes associated with the development of TRAIL resistance in (originally TRAIL-sensitive) MCL cells in order to identify such a cellular 'weakness' of TRAIL-resistant MCL cells. To identify the specific protein expression changes in the TRAIL-resistant cells, we derived a TRAIL-resistant HBL-2 subclone (HBL-2/R) from the originally TRAIL-sensitive HBL-2 cell line, and performed differential analysis of the surface expression of TRAIL receptors and comparative proteomic analysis of the HBL-2/R and HBL-2 cells.

TRAIL-resistant cell line. The TRAIL-resistant HBL-2 subclone (HBL-2/R) was derived from the originally TRAIL-sensitive HBL-2 cell line by selective pressure of increasing TRAIL concentration in medium over 5 weeks. While the $\mathrm{IC}_{50}$ for TRAIL in the originally sensitive HBL-2 cells was $1 \mathrm{ng} / \mathrm{ml}$ at $48 \mathrm{~h}$ (data not shown), the resulting HBL-2/R subclone proliferated in up to $1,000 \mathrm{ng} / \mathrm{ml}$ TRAIL concentration in medium and was therefore $>1,000$-fold more resistant to TRAIL than the HBL-2 cells (Fig. 1).

TRAIL receptors - flow cytometric analysis of cell surface expression. The attenuated expression of TRAIL death receptors DR4 and DR5 has been previously described as a cause of TRAIL resistance. We therefore determined the relative expression of TRAIL receptors in HBL-2 and HBL-2/R cells by flow cytometry (Fig. 2). The expression of DR4, DR5, DcR1 and DcR2 in the HBL-2/R cells was markedly decreased compared to the HBL-2 cells. The marked downregulation of death receptors DR4 and DR5 explains the resistance of the HBL-2/R cells to TRAIL, while the downregulation of decoy receptors DcR1 and DcR2 may indicate further, more complex phenotypic changes in the HBL-2/R cells. 


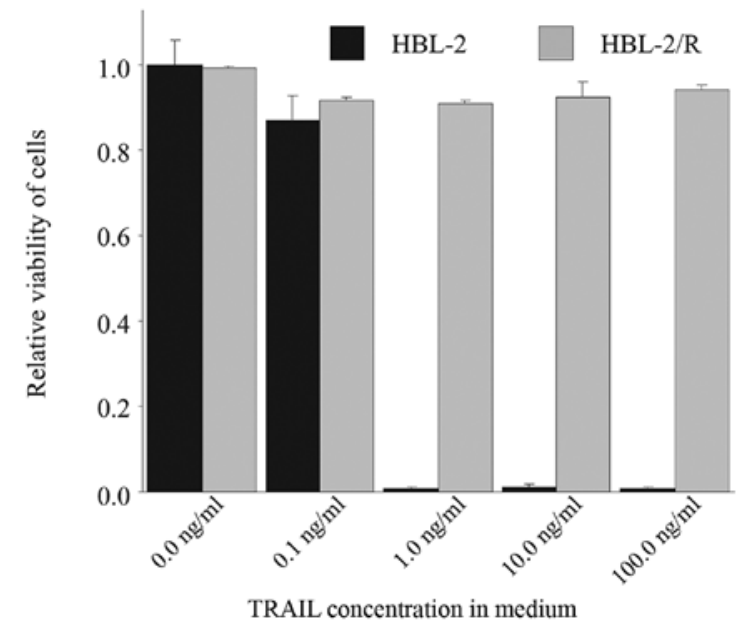

Figure 1. Relative cytotoxicity of TRAIL. Viability of TRAIL-sensitive HBL-2 cells and TRAIL-resistant HBL-2/R cells after $78 \mathrm{~h}$ in medium with recombinant TRAIL was determined by WTS-based colorimetric assay. Absorbance value of HBL-2 cells grown in medium without TRAIL was set to 1 .

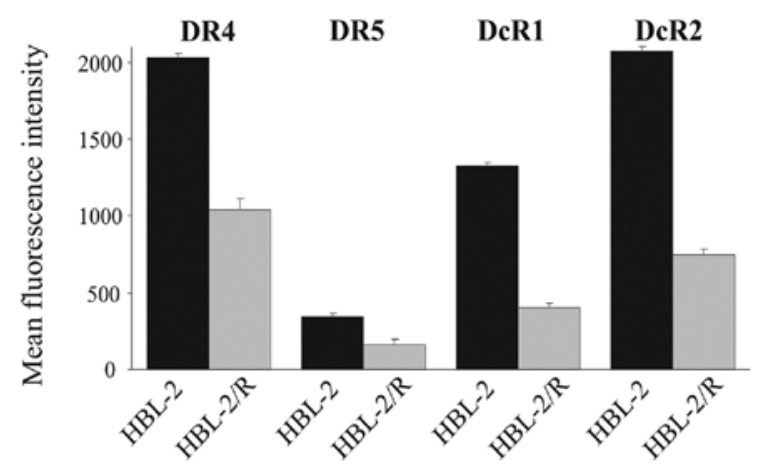

Figure 2. Cell surface expression of TRAIL receptors. HBL-2 and HBL-2/R cells were labeled with phycoerythrin-conjugated antibodies against the TRAIL cell surface receptors, DR4, DR5, DcR1 and DcR2, and the expression of the receptors was analyzed by flow cytometry. Cells without staining and isotype controls served as the blank controls.

Proteomic analysis. In order to identify specific changes in protein expression associated with TRAIL resistance in HBL-2/R cells, we performed comparative proteomic analysis of cellular homogenates of HBL-2/R and TRAIL-sensitive HBL-2 cells. Using two-dimensional electrophoresis of total cell lysates, we reproducibly detected 820 protein spots on Coomassie Brilliant Blue-stained gels. We found 21 protein spots to be significantly quantitatively changed (upregulated or downregulated, change $>1.5$-fold; $\mathrm{p}<0.05$ ) in HBL-2/R cells (Fig. 3). Using MALDI-TOF/ TOF mass spectrometry we identified all 21 proteins differentially expressed in HBL-2/R cells (Table I).

Functional annotations of the identified differentially expressed proteins were analyzed using the Kyoto Encyclopedia of Genes and Genomes (KEGG) database. Among the 21 identified proteins we found molecules involved in diverse functions, including cytoskeleton regulation, ribosome synthesis and maturation, RNA metabolism, chromosome translocation, DNA repair and replication, as well as protein folding. However, one pathway was markedly enriched in our set (hsa00230 purine metabolism) represented by 3 differentially expressed proteins. These 3 molecules are key enzymes of the purine
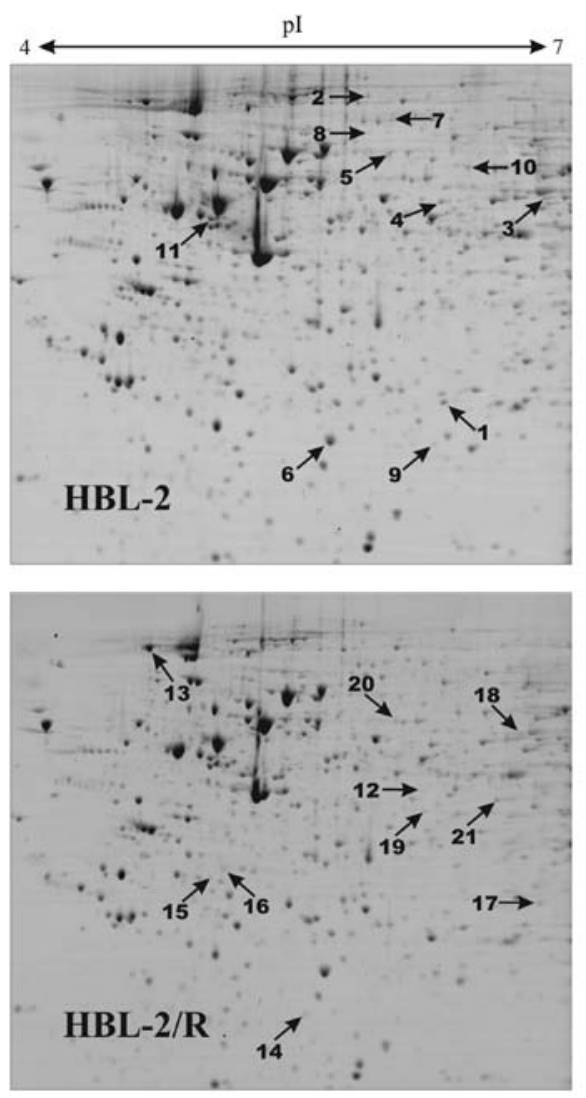

Figure 3. Two-dimensional electrophoresis of HBL-2 and HBL-2/R cells was performed on 24-cm gel strips, $\mathrm{pH}$ 4.0-7.0, 10\% SDS-PAGE. Proteins were stained with Coomassie Brilliant Blue. Differentially expressed proteins are indicated by numbered arrows (spots 1-11 indicate downregulated proteins in HBL-2/R cells, and spots 12-21 indicate upregulated proteins in HBL-2/R cells).

nucleotide metabolism (Fig. 5) and all 3 are downregulated in TRAIL-resistant HBL-2/R cells [PNP (downregulated 1.6-fold in HBL-2/R cells), APRT (downregulated 2.2-fold in HBL-2/R cells) and inosine-5'-monophosphate dehydrogenase 2 (IMPDH2, downregulated 1.6-fold in HBL-2/R cells)].

Verification of proteomic analysis. To confirm the results of proteomic analysis by an independent method we verified the decreased expression of the 2 proteins involved in purine metabolism, namely PNP and APRT, by western blot analysis in HBL-2 and HBL-2/R cell lysates (Fig. 4).

\section{Discussion}

The downregulation of the 3 key enzymes of purine metabolism can have a profound effect on nucleotide homeostasis in TRAIL-resistant lymphoma cells. Purine nucleotides, the building blocks for synthesis of DNA, RNA and enzyme co-factors, are recruited either from de novo purine synthesis from low molecular weight precursors or by recycling of free nucleobases in the so-called salvage pathway. Both pathways lead to the production of nucleoside-5'-phosphates (Fig. 5). Both pathways can supply cellular demand independently; however, their importance in different tissues is variable. In leukemic and lymphoma cells the salvage pathway is considered the major source of purine nucleotides $(30,31)$. 
Table I. List of proteins differentially expressed in HBL-2/R cells (difference at least 1.5-fold and statistical significance $\mathrm{p}<0.05$ ).

\begin{tabular}{|c|c|c|c|c|c|}
\hline $\begin{array}{l}\text { Spot } \\
\text { no. }\end{array}$ & $\begin{array}{c}\text { Swiss-Prot } \\
\text { no. }\end{array}$ & Protein name & $\begin{array}{c}\text { Fold } \\
\text { change }\end{array}$ & $\begin{array}{l}\text { Mascot } \\
\text { score }^{b}\end{array}$ & $\begin{array}{l}\text { Sequence } \\
\text { cov. }(\%)^{\mathrm{c}}\end{array}$ \\
\hline
\end{tabular}

Proteins upregulated in HBL-2/R cells

\begin{tabular}{|c|c|c|c|c|c|c|}
\hline 1 & P04792 & Heat shock protein $\beta-1$ & 3.9 & 84 & 51 & 22826 \\
\hline 2 & P42704 & Leucine-rich PPR motif-containing protein, mitochondrial & 2.6 & 100 & 23 & 159003 \\
\hline 3 & O75351 & Vacuolar protein sorting-associated protein 4B & 2.6 & 171 & 32 & 49443 \\
\hline 4 & P23381 & Tryptophanyl-tRNA synthetase, cytoplasmic & 2.4 & 240 & 54 & 53474 \\
\hline 5 & P20591 & Interferon-induced GTP-binding protein Mx1 & 2.2 & 176 & 42 & 75872 \\
\hline 6 & P09211 & Glutathione S-transferase P & 1.9 & 110 & 56 & 23569 \\
\hline 7 & P06396 & Gelsolin & 1.9 & 115 & 22 & 86043 \\
\hline 8 & P13010 & $\mathrm{X}$-ray repair cross-complementing protein 5 & 1.7 & 262 & 46 & 83222 \\
\hline 9 & Q9HAV7 & GrpE protein homolog 1, mitochondrial & 1.6 & 99 & 44 & 24492 \\
\hline 10 & $\mathrm{O} 43776$ & Asparaginyl-tRNA synthetase, cytoplasmic & 1.5 & 250 & 41 & 63758 \\
\hline 11 & Q15084 & Protein disulfide-isomerase A6 & 1.5 & 76 & 29 & 48490 \\
\hline \multicolumn{7}{|c|}{ roteins downregulated in HBL-2/R cells } \\
\hline 12 & P08559 & Pyruvate dehydrogenase E1 component subunit $\alpha$ & 3.2 & 111 & 32 & 43952 \\
\hline 13 & P19338 & Nucleolin & 2.4 & 146 & 29 & 76625 \\
\hline 14 & P07741 & Adenine phosphoribosyltransferase & 2.2 & 227 & 79 & 19766 \\
\hline 15 & O75792 & Ribonuclease $\mathrm{H} 2$ subunit A & 1.7 & 348 & 72 & 33716 \\
\hline 16 & Q07955 & Serine/arginine-rich splicing factor 1 & 1.7 & 82 & 35 & 27842 \\
\hline 17 & P00491 & Purine nucleoside phosphorylase & 1.6 & 182 & 68 & 32325 \\
\hline 18 & P12268 & Inosine-5'-monophosphate dehydrogenase 2 & 1.6 & 230 & 44 & 56226 \\
\hline 19 & P40121 & Macrophage-capping protein & 1.6 & 102 & 41 & 38760 \\
\hline 20 & P13674 & Prolyl 4-hydroxylase subunit $\alpha-1$ & 1.5 & 234 & 48 & 61296 \\
\hline 21 & Q15019 & Septin-2 & 1.5 & 62 & 26 & 41689 \\
\hline
\end{tabular}

${ }^{\text {aS }}$ wiss-Prot no. is the code under which the identified protein is deposited in the Swiss-Prot database. ${ }^{b}$ Mascot score helps to estimate the correctness of the individual hit. It is expressed as $-10 \mathrm{x} \log (\mathrm{P})$ where $\mathrm{P}$ is the probability that the observed match is a random event. ${ }^{c} \mathrm{Sequence}$ coverage is the number of amino acids spanned by the assigned peptides divided by the sequence length.

A

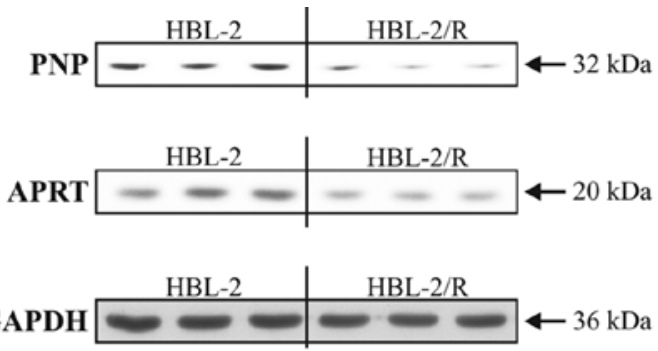

B

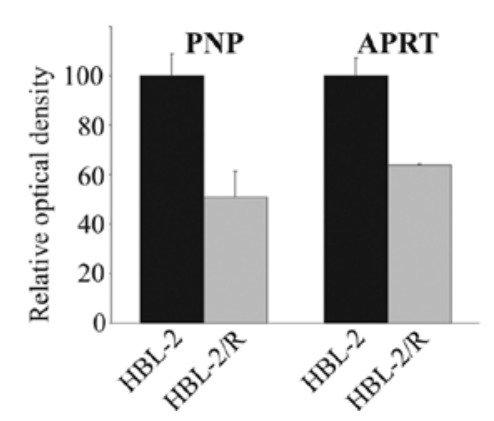

Figure 4. Relative expression of purine nucleoside phosphorylase (PNP) and adenine phosphoribosyltransferase (APRT) in HBL-2 and HBL-2/R cell lysates determined by western blot analysis. (A) Triplicate cell lysates were separated on 12\% SDS-PAGE minigels. Proteins were then transferred onto PVDF membranes, blocked and probed with either anti-APRT, or anti-PNP antibody. Anti-GAPDH antibody was used as the loading control. The bands were visualized by HRP-conjugated secondary antibodies. (B) The values of integrated optical densities of PNP and APRT in HBL-2 cells were set to 100.

The de novo synthesis of purine nucleotides requires 5-phosphoribosyl-1-pyrophosphate (PRPP), ATP, glutamine, glycine, $\mathrm{CO}_{2}$, aspartate and formate to create the first fully formed nucleotide, inosine-5'-monophosphate (IMP). IMP represents a branch point for purine biosynthesis, since it can be converted either to guanosine-5'-monophosphate (GMP) by IMPDH2 (downregulated in HBL-2/R cells) or to adenosine5'-monophosphate (Fig. 5).
The catabolism of purine nucleotides leads to the liberation of free purine bases by PNP (downregulated in HBL-2/R cells). In the salvage pathway the free bases are reconverted back to nucleoside-5'-monophosphates in a reaction with activated sugar (PRPP) catalyzed by APRT (downregulated in HBL-2/R cells) or hypoxanthine-guanine phosphoribosyltransferase (32) (Fig. 5). Ribonucleotides are converted by ribonucleotide reductase into the corresponding deoxyribonucleotides. 


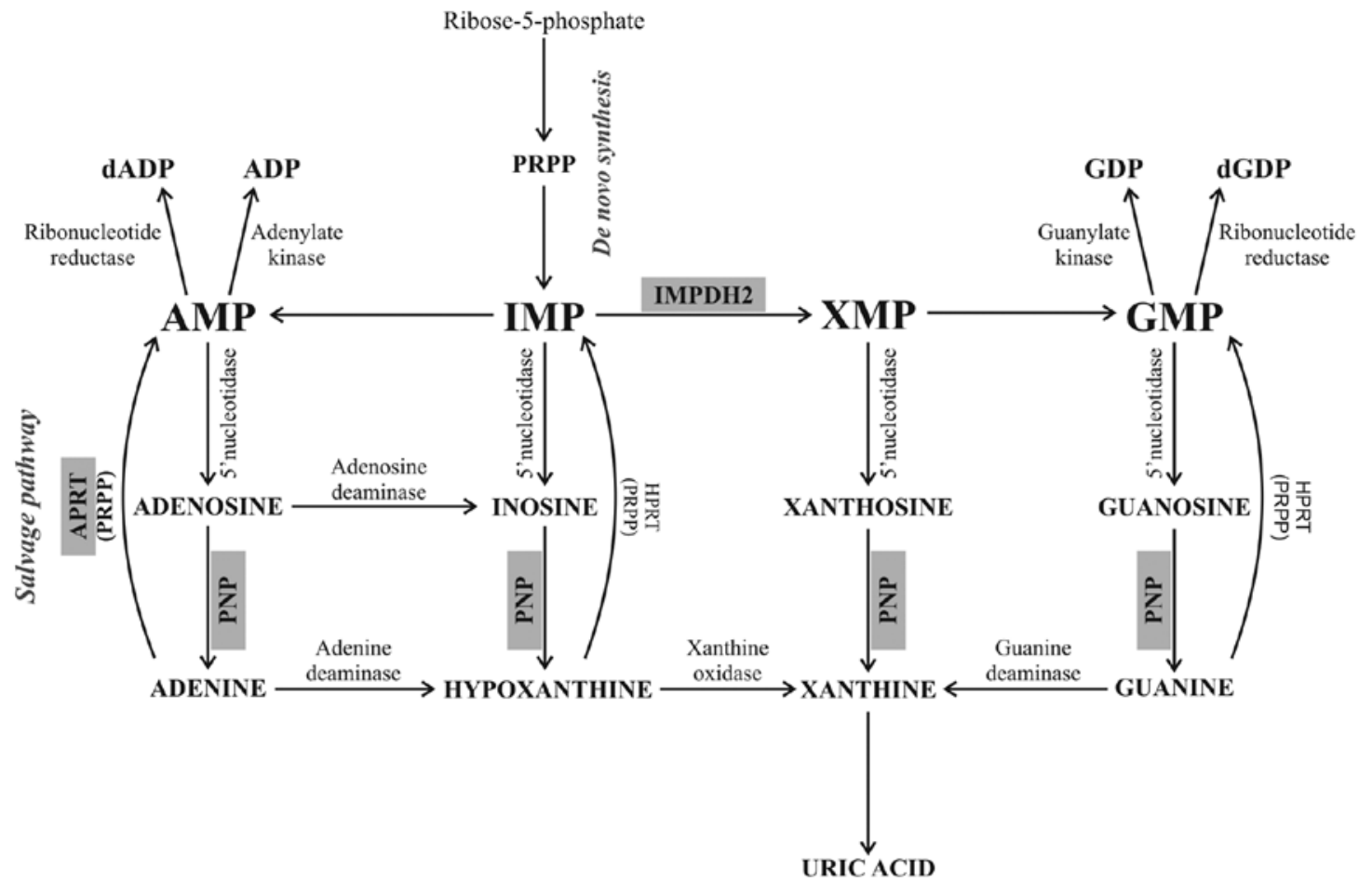

Figure 5. Scheme of the purine metabolism pathways, showing the position of IMPDH2, APRT and PNP in purine nucleotide biosynthesis, adopted from a previous study (35). The de novo synthesis of purine nucleotides begins with the phosphorylation of ribose-5-phosphate to form PRPP. In a number of reactions, PRPP creates the first fully formed nucleotide, IMP. IMP is converted by IMPDH2 to GMP. PNP catalyzes the reversible cleavage of purine nucleosides, releasing purine nucleobases (adenine, hypoxanthine, xanthine and guanine). In the salvage pathway the free nucleobases can be reconverted back to nucleoside-5'-monophosphates in a reaction with activated sugar (PRPP) catalyzed by APRT. IMPDH2, inosine-5'-monophosphate dehydrogenase 2; APRT, adenine phosphoribosyltransferase; PNP, purine nucleoside phosphorylase; PRPP, 5-phosphoribosyl-1-pyrophosphate; IMP, inosine-5'-monophosphate; GMP, guanosine-5'-monophosphate; dADP, deoxyadenosine diphosphate; ADP, adenosine diphosphate; GDP, guanosine diphosphate; dGDP, deoxyguanosine diphosphate; AMP, adenosine monophosphate; XMP, xanthosine monophosphate.

The delicate balance of enzyme activities and concentrations of products and intermediates are critical for purine (nucleotide) homeostasis. The inhibition of PNP results in the accumulation of its substrate, 2'-deoxyguanosine which is further phosphorylated to deoxyguanosine triphosphate (dGTP). A high intracellular concentration of dGTP inhibits cell proliferation and induces apoptosis (33-35). If APRT is inhibited, accumulated adenine is oxidized to insoluble 2,8-dihydroxyadenine. Accumulation of this precipitate results in cell death (32). Similarly, the inhibition of IMPDH2 leads to depletion of guanosine nucleotides, which blocks DNA synthesis and cell division $(36,37)$.

Disruption of the purine nucleotide metabolism generally results in an accumulation and/or a lack of ribonucleotides or deoxyribonucleotides or metabolic intermediates with potentially cytotoxic consequences. The observed decreased expression of the 3 purine metabolism enzymes affects both de novo synthesis and the salvage pathway of purine metabolism and may also affect purine nucleotide homeostasis in TRAIL-resistant HBL-2/R cells. Such an imbalance may represent a selective disadvantage for the affected cells. Such a 'weakness' may not be apparent under normal circumstances but may become critical under stress or unfavorable conditions. As the proliferation rates of HBL-2/R and HBL-2 cells are comparable, the proposed imbalance in purine nucleotide metabolism in TRAIL-resistant cells is possibly mild and/ or well compensated in vitro. However, this 'weakness' may become apparent due to lack of building blocks for DNA and
RNA synthesis in the environment or upon further disruption of purine metabolism. Since both pathways of purine metabolism are compromised in TRAIL-resistant MCL cells, these cells should be vulnerable to further inactivation of purine nucleotide metabolism enzymes. Therefore, drugs that target (already disbalanced) purine metabolism should be highly cytotoxic to TRAIL-resistant cells (compared to non-malignant cells) and may therefore be selectively effective in the elimination of TRAIL-resistant MCL cells in experimental therapy. There are several approved inhibitors of purine metabolism, such as methotrexate (inhibits purine de novo synthesis via dihydrofolate reductase) (38), ribavirin and mycophenolic acid (inhibitors of IMPDH2) $(39,40)$ or forodesine (a novel inhibitor of PNP) $(41,42)$, available for clinical use.

The adaptation of cancer cells to cytostatic and cytotoxic drugs is associated to a certain degree with extensive changes in the cell phenotype. Some of the molecular changes, although seemingly unrelated to the mechanism of resistance, can provide a selective disadvantage to the cells and such a 'weakness' may be used as a potential therapeutic target. By the presented proteomic analysis of the changes associated with resistance to TRAIL in MCL HBL-2 cells, we demonstrated the downregulation of all types of TRAIL receptors and identified the altered expression of several proteins including 3 enzymes of the purine metabolism pathway. This downregulated pathway potentially represents a 'weakness' of the TRAIL-resistant MCL cells and has potential as a therapeutic target for the selective elimination of such cells in the future. 


\section{Acknowledgements}

This study was supported by the Grant Agency of Charles University (GAUK 251180111210 and 253284 700712), by the Grant Agency of the Czech Republic (305/09/1390), by the Ministry of Education, Youth and Sports (PRVOUK P24/LF1/3 and SVV 2012-264507), UNCE 204021 and the Ministry of Health of the Czech Republic (IGA MZ NT12248-5, IGA-MZ NT13201-4).

\section{References}

1. Sant M, Allemani C, Tereanu C, et al: Incidence of hematologic malignancies in Europe by morphologic subtype: results of the HAEMACARE project. Blood 116: 3724-3734, 2010.

2. Perez-Galan P,Dreyling M and Wiestner A: Mantle cell lymphoma: biology, pathogenesis, and the molecular basis of treatment in the genomic era. Blood 117: 26-38, 2011.

3. Tsujimoto Y, Yunis J, Onorato-Showe L, Erikson J, Nowell PC and Croce CM: Molecular cloning of the chromosomal breakpoint of B-cell lymphomas and leukemias with the $\mathrm{t}(11 ; 14)$ chromosome translocation. Science 224: 1403-1406, 1984.

4. Williams ME, Swerdlow SH, Rosenberg CL and Arnold A: Characterization of chromosome 11 translocation breakpoints at the bcl-1 and PRAD1 loci in centrocytic lymphoma. Cancer Res 52: 5541S-5544S, 1992.

5. Humala K and Younes A: Current and emerging new treatment strategies for mantle cell lymphoma. Leuk Lymphoma: Feb 19 2013 (Epub ahead of print).

6. Wiley SR, Schooley K, Smolak PJ, et al: Identification and characterization of a new member of the TNF family that induces apoptosis. Immunity 3: 673-682, 1995.

7. Pitti RM, Marsters SA, Ruppert S, Donahue CJ, Moore A and Ashkenazi A: Induction of apoptosis by Apo-2 ligand, a new member of the tumor necrosis factor cytokine family. J Biol Chem 271: 12687-12690, 1996.

8. Ashkenazi A and Dixit VM: Death receptors: signaling and modulation. Science 281: 1305-1308, 1998.

9. Sheridan JP, Marsters SA, Pitti RM, et al: Control of TRAILinduced apoptosis by a family of signaling and decoy receptors. Science 277: 818-821, 1997.

10. Ashkenazi A: Targeting death and decoy receptors of the tumournecrosis factor superfamily. Nat Rev Cancer 2: 420-430, 2002.

11. Castro Alves C, Terziyska N, Grunert M, et al: Leukemiainitiating cells of patient-derived acute lymphoblastic leukemia xenografts are sensitive toward TRAIL. Blood 119: 4224-4227, 2012.

12. Peter ME and Krammer PH: The CD95(APO-1/Fas) DISC and beyond. Cell Death Differ 10: 26-35, 2003.

13. Spierings DC: Tissue distribution of the death ligand TRAIL and its receptors. J Histochem Cytochem 52: 821-831, 2004.

14. Petrak J, Toman O, Simonova T, et al: Identification of molecular targets for selective elimination of TRAIL-resistant leukemia cells. From spots to in vitro assays using TOP15 charts. Proteomics 9: 5006-5015, 2009.

15. Molinsky J, Klanova M, Koc M, et al: Roscovitine sensitizes leukemia and lymphoma cells to tumor necrosis factorrelated apoptosis-inducing ligand-induced apoptosis. Leuk Lymphoma 54: 372-380, 2013.

16. Klener P, Leahomschi S, Molinsky J, et al: TRAIL-induced apoptosis of HL60 leukemia cells: two distinct phenotypes of acquired TRAIL resistance that are accompanied with resistance to TNFalpha but not to idarubicin and cytarabine. Blood Cells Mol Dis 42: 77-84, 2009.

17. Leahomschi S, Molinsky J, Klanova M, et al: Multi-level disruption of the extrinsic apoptotic pathway mediates resistance of leukemia cells to TNF-related apoptosis-inducing ligand (TRAIL). Neoplasma 60: 223-231, 2013.

18. Wen J, Ramadevi N, Nguyen D, Perkins C, Worthington E and Bhalla K: Antileukemic drugs increase death receptor 5 levels and enhance Apo-2L-induced apoptosis of human acute leukemia cells. Blood 96: 3900-3906, 2000.

19. Plasilova M, Zivny J, Jelinek J, et al: TRAIL (Apo2L) suppresses growth of primary human leukemia and myelodysplasia progenitors. Leukemia 16: 67-73, 2002.
20. Ashkenazi A, Pai RC, Fong S, et al: Safety and antitumor activity of recombinant soluble Apo2 ligand. J Clin Invest 104: 155-162, 1999.

21. Di Pietro R and Zauli G: Emerging non-apoptotic functions of tumor necrosis factor-related apoptosis-inducing ligand (TRAIL)/ Apo2L. J Cell Physiol 201: 331-340, 2004.

22. Kelley SK, Harris LA, Xie D, et al: Preclinical studies to predict the disposition of Apo2L/tumor necrosis factor-related apoptosisinducing ligand in humans: characterization of in vivo efficacy, pharmacokinetics, and safety. J Pharmacol Exp Ther 299: 31-38, 2001.

23. Lawrence D, Shahrokh Z, Marsters S, et al: Differential hepatocyte toxicity of recombinant Apo2L/TRAIL versions. Nat Med 7: 383-385, 2001

24. Roth W, Isenmann S, Naumann U, et al: Locoregional Apo2L/ TRAIL eradicates intracranial human malignant glioma xenografts in athymic mice in the absence of neurotoxicity. Biochem Biophys Res Commun 265: 479-483, 1999.

25. Walczak H, Miller RE, Ariail K, et al: Tumoricidal activity of tumor necrosis factor-related apoptosis-inducing ligand in vivo. Nat Med 5: 157-163, 1999.

26. Hylander BL, Pitoniak R, Penetrante RB, et al: The anti-tumor effect of Apo2L/TRAIL on patient pancreatic adenocarcinomas grown as xenografts in SCID mice. J Transl Med 3: 22, 2005.

27. Dimberg LY, Anderson CK, Camidge R, Behbakht K, Thorburn A and Ford HL: On the TRAIL to successful cancer therapy? Predicting and counteracting resistance against TRAIL-based therapeutics. Oncogene: 14 May, 2012 (Epub ahead of print). doi: 10.1038/onc.2012.164, 2012.

28. Zhang L and Fang B: Mechanisms of resistance to TRAIL-induced apoptosis in cancer. Cancer Gene Ther 12: 228-237, 2005.

29. Maksimovic-Ivanic D, Stosic-Grujicic S, Nicoletti F and Mijatovic S: Resistance to TRAIL and how to surmount it. Immunol Res 52: 157-168, 2012.

30. Scavennec J, Maraninchi D, Gastaut JA, Carcassonne Y and Cailla HL: Purine and pyrimidine ribonucleoside monophosphate patterns of peripheral blood and bone marrow cells in human acute leukemias. Cancer Res 42: 1326-1330, 1982.

31. Natsumeda Y, Prajda N, Donohue JP, Glover JL and Weber G: Enzymic capacities of purine de novo and salvage pathways for nucleotide synthesis in normal and neoplastic tissues. Cancer Res 44: 2475-2479, 1984.

32. Bollee G, Harambat J, Bensman A, Knebelmann B, Daudon M and Ceballos-Picot I: Adenine phosphoribosyltransferase deficiency. Clin J Am Soc Nephrol 7: 1521-1527, 2012.

33. Bantia S, Miller PJ, Parker CD, et al: Purine nucleoside phosphorylase inhibitor BCX-1777 (Immucillin-H) - a novel potent and orally active immunosuppressive agent. Int Immunopharmacol 1: 1199-1210, 2001.

34. Bantia S, Montgomery JA, Johnson HG and Walsh GM: In vivo and in vitro pharmacologic activity of the purine nucleoside phosphorylase inhibitor BCX-34: the role of GTP and dGTP. Immunopharmacology 35: 53-63, 1996.

35. Galmarini CM, Popowycz F and Joseph B: Cytotoxic nucleoside analogues: different strategies to improve their clinical efficacy. Curr Med Chem 15: 1072-1082, 2008.

36. Allison AC and Eugui EM: Mycophenolate mofetil and its mechanisms of action. Immunopharmacology 47: 85-118, 2000.

37. Hedstrom L: IMP dehydrogenase: structure, mechanism, and inhibition. Chem Rev 109: 2903-2928, 2009.

38. Fairbanks LD, Ruckemann K, Qiu Y, et al: Methotrexate inhibits the first committed step of purine biosynthesis in mitogen-stimulated human T-lymphocytes: a metabolic basis for efficacy in rheumatoid arthritis? Biochem J 342: 143-152, 1999.

39. Allison AC and Eugui EM: The design and development of an immunosuppressive drug, mycophenolate mofetil. Springer Semin Immunopathol 14: 353-380, 1993.

40. Zhou S, Liu R, Baroudy BM, Malcolm BA and Reyes GR: The effect of ribavirin and IMPDH inhibitors on hepatitis $C$ virus subgenomic replicon RNA. Virology 310: 333-342, 2003.

41. Gandhi V, Kilpatrick JM, Plunkett W, et al: A proof-of-principle pharmacokinetic, pharmacodynamic, and clinical study with purine nucleoside phosphorylase inhibitor immucillin-H (BCX-1777, forodesine). Blood 106: 4253-4260, 2005.

42. Miles RW, Tyler PC, Furneaux RH, Bagdassarian CK and Schramm VL: One-third-the-sites transition-state inhibitors for purine nucleoside phosphorylase. Biochemistry 37: 8615-8621, 1998. 